\title{
Astrometric Microlensing by Finite-size Lenses
}

\author{
Rohta Takahashi \\ Yukawa institute for theoretical physics, Kyoto University, Sakyo-ku, \\ Kyoto 606-8502, Japan
}

\begin{abstract}
I have calculated astrometrical trajectories of microlensing phenomena caused by a spherical finite-size lens consisting of opaque material. For details, see Takahashi (2003).
\end{abstract}

\section{Microlens Trajectories and Observational Feasibility}

Thanks to the revolutionary progress in the technique of astrometrical observation, a realistic possibility has emerged of elucidating the nature of baryonic dark matter (e.g. MACHOs, small cold clouds) from the centroidal image trajectory of the microlens phenomena. To check if the size of lens objects can be determined by astrometrical observation, I have calculated astrometrical trajectories of microlensing phenomena caused by a spherical finite-size lens consisting of opaque material, and found that these trajectories can be classified into three types according to occultation of the plus and minus image. All three types of trajectories have distinct features which reflect the effects of a finite-size lens. From these trajectories, the angular radius of the lens can be determined for two types of trajectories, and from the third type of trajectory the upper and the lower limits of the angular radius can be determined, if these features can be measured. I have investigated finite-lens effects in nearby high-proper-motion stars identified in Salim \& Gould (2000) which could cause observable astrometric microlensing events during SIM's lifetime. None of them shows a finite-lens effect with $10 \mu$ arcsecond positional accuracy, one event shows finite-lens effects with $1 \mu$ arcsecond positional accuracy, and 6 events show finite-lens effects with $0.1 \mu$ arcsecond positional accuracy.

Calculations of microlensing trajectories for point mass hold for any spherical lens of finite size, which is a conclusion from Birkhoff's theorem, so long as the photon trajectories do not pass inside the lens where there is some degree of opacity, in which case the photons would not arrive at an observer. The time variation of the angular $\theta_{x}$ and $\theta_{y}$ coordinates of astrometrical trajectories and trajectories are shown in figure 1 for the three trajectory types $\mathrm{A}($ top $), \mathrm{B}($ middle) and $\mathrm{C}($ bottom $)$.

\section{References}

Salim, S. \& Gould, A. 2000, ApJ, 539, 241

Takahashi, R. 2003, ApJ, 595, 418 

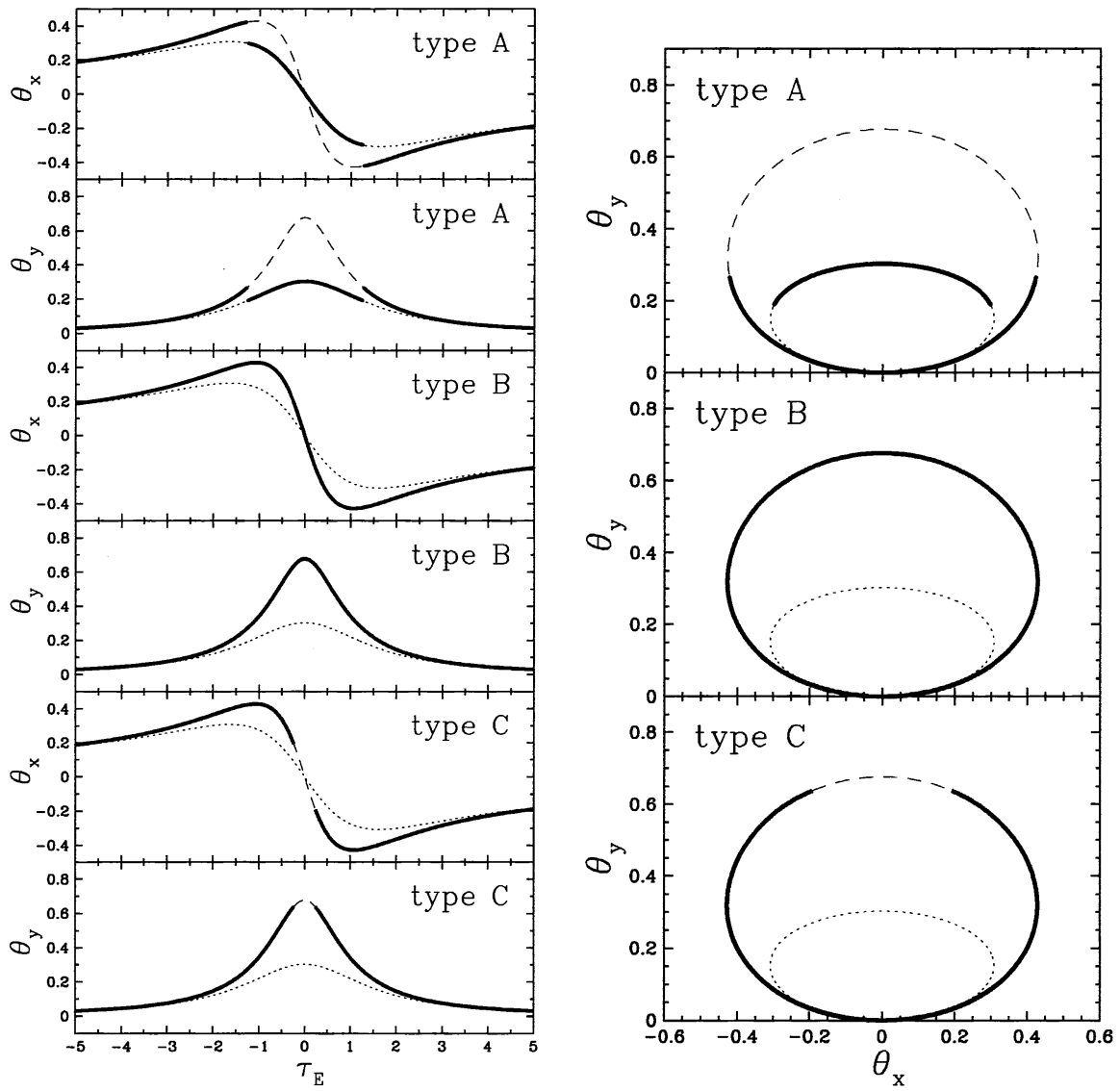

Figure 1. Right figures show the $\theta_{x}$ and $\theta_{y}$ coordinates, $\left(\theta_{x}, \theta_{y}\right)$, of microlens trajectories as a function of time for image trajectories (thick lines) of three types, $\mathrm{A}, \mathrm{B}$ and $\mathrm{C}$. The $\theta_{x}$ and $\theta_{y}$ coordinates, $\left(\theta_{x}, \theta_{y}\right)$, for trajectories of the centroidal images (dotted lines) and the plus images (dashed lines) for a point lens are shown. Left figures shows astrometric microlens trajectories of three types, A, B and C (thick lines) for a finite-size lens. The trajectories of the centroidal images (dotted lines) and the plus images (dashed lines) for a point lens are shown. 